\title{
Two further cases of Graves' disease following SARS-Cov-2 vaccination
}

\author{
G. Zettinig' ${ }^{1} \cdot$ M. Krebs ${ }^{2}$
}

Received: 20 July 2021 / Accepted: 22 July 2021 / Published online: 3 August 2021

(C) Italian Society of Endocrinology (SIE) 2021

Keywords SARS-Cov-2 RNA vaccination · Graves' disease · COVID · Thyroid

\section{Dear Editor,}

Recently, two cases of Graves' disease after SARS-Cov2 RNA vaccination with tozinameran have been reported [1]. Here, we report two further cases: a late recurrence after 17 years and an initial manifestation.

A 71-year-old lady had subtotal resection of the left lobe. Soon thereafter, Graves' disease occurred, treated by thyreostatic drugs until 2004. She has been in care of our Thyroid Center since 2008. She was euthyroid with normal TSH-receptor-antibody (TRAB) levels at yearly checkups. Sonography also did not show any dynamics: an enlarged $20 \mathrm{ml}$ right lobe and a small $4 \mathrm{ml}$ left lobe after partial resection. The parenchyma was slightly hypoechogenic. She was vaccinated with tozinameran on 21. March and 11. April. From Mid-May on, she noticed palpitations and sweating. On 26th May, she was seen in our Thyroid Center with a free $\mathrm{T} 4 \mathrm{of} 3.56 \mathrm{ng} / \mathrm{dl}$ (normal $0.70-1.70$ ), a free T3 of $11.10 \mathrm{pg} /$ $\mathrm{ml}$ (normal 2.15-4.12), and a TRAB level of 4.2 IU/l (normal $<1.5$ ). A marked change in sonography with multiple confluent anechogenic areas and increased vascularization was observed. Scintigraphy revealed the small (partly resected) left lobe and the enlarged right lobe with a patchy inhomogenous tracer distribution. Uptake was only mildly increased. Thyreostatic treatment led to a rapid normalization of thyroid function.

G. Zettinig

georg.zettinig@meduniwien.ac.at

M. Krebs

michael.krebs@meduniwien.ac.at

1 Vienna Thyroid Center Schilddruesenpraxis Josefstadt, Laudongasse 12-8, 1080 Vienna, Austria

2 Department of Endocrinology and Metabolism, Medical University Vienna, Waehringer Guertel 18-20, 1090 Vienna, Austria
Patient 2 is a 46-year-old male without previous history of thyroid disease. Since the age of 43 , regular blood tests documented euthyroidism. He had his first vaccination with tozinameran on 6. May. A routine blood test on 21. May noticed hyperthyroidism. At his first visit at our Thyroid Center on 1. June, he had a free T4 of 1.63 , a free T3 of $5.18 \mathrm{pg} / \mathrm{ml}$, and a TRAB level of 2.9 IU/l. Sonography showed a slightly enlarged thyroid. In the hypoechogenic parenchyma, large anechogenic areas with increased vascularization were seen. Scintigraphy also showed a patchy, very inhomogenous $\mathrm{Tc} 99 \mathrm{~m}$ accumulation, and the uptake was normal. Thyreostatic treatment also led to a rapid normalization of thyroid function.

Both patients showed a rather unusual inhomogenous scintigraphic pattern: corresponding to the areas with the highest Tc99m accumulation, sonography showed markedly hypoechoic areas.

A possible interaction between the autoimmune system and SARS-Cov2 RNA vaccination is still a matter of discussion. In SARS-Cov2-related atypical thyroiditis, focal markedly hypoechoic areas were published with corresponding focal reduced Tc99m uptake [2]. Reports on Graves' disease after COVID-19 in some patients indicate that SARS-Cov2 could play a role in the induction of latent or new-onset thyroid autoimmunity [3]. In the first report on Graves' disease after SARS-Cov-2 RNA vaccination ASIA (autoimmune/inflammatory syndrome induced by adjuvants) was suggested as a cause [1]. Not only a random coincidence or ASIA could have induced autoimmune hyperreactivity in our patients, but also the vaccine itself could have triggered autoimmune thyroid disease. Our findings further indicate that in the ongoing phase 3 trials, thyroid autoimmunity should be in the focus of the investigators.

Author contributions GZ concept and design, data acquisition, analysis and interpretation of the data, and drafting the manuscript. MK concept 
and design, interpretation of the data, and critical review. All authors have reviewed and approved the article before submission and agree to be accountable for all aspects of the work in ensuring that questions related to the accuracy or integrity of any part of the work are appropriately investigated and resolved.

Funding This research did not receive any specific grant from any funding agency in the public, commercial or not-for-profit sector.

Data availability All data and materials support their published claims and comply with field standards.

\section{Declarations}

Conflict of interest No competing interests, no personal financial interests, no funding, and no employment by an organization that may gain or lose financially from publication of the article. There are also no other competing interests regarding the manuscript.

Ethical approval All procedures performed during this retrospective study were in accordance with the ethical standards of institutional and/ or national research committee and with the 1964 Helsinki Declaration and its later amendments or comparable ethical standards. The ethical committee approval is not required for case reports.

Consent to participate Informed consent was obtained from both patients.
Consent for publication We thank the patients for granting permission to publish this information.

\section{References}

1. Vera-Lastra O, Ordinola Navarro A, Cruz Domiguez MP, Medina G, Sánchez Valadez TI, Jara LJ (2021) Two cases of Graves' disease following SARS-CoV-2 vaccination: an autoimmune/inflammatory syndrome induced by adjuvants. Thyroid. https://doi.org/ 10.1089/thy.2021.0142 (In press)

2. Muller I, Cannavaro D, Dazzi D, Covelli D, Mantovani G, Muscatello A, Ferrante E, Orsi E, Resi V, Longari V et al (2020) SARSCoV-2-related atypical thyroiditis. Lancet Diabetes Endocrinol 8:739-741. https://doi.org/10.1016/S2213-8587(20)30266-7

3. Jiménez-Blanco S, Pla-Peris B, Marazuela M (2021) COVID-19: a cause of recurrent Graves' hyperthyroidism? J Endocrinol Invest 44:387-388. https://doi.org/10.1007/s40618-020-01440-0

Publisher's Note Springer Nature remains neutral with regard to jurisdictional claims in published maps and institutional affiliations. 